\title{
ON THE EXTREMAL SOLUTIONS OF $n$ TH-ORDER LINEAR DIFFERENTIAL EQUATIONS
}

\author{
W. J. KIM
}

ABSTRACT. Distribution of zeros of extremal solutions of linear $n$ th-order differential equations is discussed. Existence and nonexistence of extremal solutions with certain zero distributions are established. For instance, it is proved that every extremal solution for $\left[\alpha, \eta_{1}(\alpha)\right]$ of the equation $y^{(n)}+p_{n-1} y^{(n-1)}+\cdots+p_{0} y=0$ has a zero of order 2 at $\eta_{1}(\alpha)$ and has no more than $n-2$ zeros on $\left[x, \eta_{1}(\alpha)\right)$ if $p_{i} \leqq 0, i=0,1, \cdots, n-2$.

1. Introduction. We shall consider the $n$ th-order differential equation

$$
y^{(n)}+p_{n-1} y^{(n-1)}+\cdots+p_{0} y=0,
$$

where $p_{0}, p_{1}, \cdots, p_{n-1}$ are real-valued functions which are continuous on an interval $I$. A solution $y$ of (1.1) is said to have a zero of order $k$ at $\zeta$ if $y(\zeta)=y^{\prime}(\zeta)=\cdots=y^{(k-1)}(\zeta)=0$; if further $y^{(k)}(\zeta) \neq 0$, we say that $y$ has a zero of order exactly $k$ at $\zeta$. The first conjugate point $\eta_{1}(\alpha)$ of a point $\alpha \in I$ is the smallest number $\beta^{\prime}>\alpha, \beta \in I$, such that there exists a nontrivial solution of (1.1) which vanishes at $\alpha$ and has $n$ zeros (where the zeros are counted with their multiplicities) on $[\alpha, \beta]$. A nontrivial solution of (1.1) which has $n$ zeros on $\left[\alpha, \eta_{1}(\alpha)\right]$ is called an extremal solution for the interval $\left[\alpha, r_{i 1}(\alpha)\right]$. Equation (1.1) is said to be disconjugate on $I$ if $\eta_{1}(\alpha) \notin I$ for all $\alpha \in I$. If (1.1) is not disconjugate on $I$, it is well known that $\eta_{1}(\alpha) \in I$ for some $\alpha \in I$ and that there is an extremal solution of (1.1) for $\left[\alpha, \eta_{1}(\alpha)\right]$ which does not vanish on $\left(\alpha, \eta_{1}(\alpha)\right)$ [14]. A nontrivial solution of (1.1) is said to have an $i_{1}-i_{2}-\cdots-i_{m}$ distribution of zeros on $I$ if it has a zero of order $i_{k}$ at $x_{k} \in I, x_{1}<x_{2}<$ $\cdots<x_{m}, k=1,2, \cdots, m$.

Suppose that the third-order equation

$$
y^{\prime \prime \prime}+p_{2} y^{\prime \prime}+p_{1} y^{\prime}+p_{0} y=0
$$

has a nontrivial solution with three zeros on $I$. Then it is well known that (1.2) has a solution with a 1-2 or 2-1 distribution of zeros on $I$ [2], [3], [4]. Results of a similar nature for the fourth-order equation have been

Received by the editors April 24, 1971.

AMS 1969 subject classifications. Primary 3442, 3420.

Key words and phrases. nth-order linear equations with real-valued continuous coefficients, zero distribution of extremal solutions, existence and nonexistence.

(c) American Matnematical Society 1972 
obtained by Aliev [1] and Peterson [11]. Distribution of zeros of solutions of $n$ th-order linear differential equations has been studied by Hartman [5], Sherman [14], [15], and others [7], [10], [12]. For a more complete review of related results, see [4], [9].

Disconjugacy is intimately connected with various aspects of the qualitative theory of (1.1). For example, disconjugacy implies the existence of a Pólya-Mammana expansion, which in turn establishes certain integral inequalities. However, a closer examination reveals that the disconjugacy is not necessary; the nonexistence of solutions with certain zero distribution suffices to establish the integral inequalities. This is but one example of what makes meaningful the study of zero distributions.

In this paper we shall be concerned with the distribution of zeros of extremal solutions of (1.1).

In $\S 2$ we establish results concerning the existence of extremal solutions with certain zero distributions. For instance, we prove: If (1.1) has an extremal solution with an $i_{1}-i_{2}-\cdots-i_{l-1}-1$ distribution of zeros, then it also has an extremal solution with a $j_{1}-j_{2}-\cdots-j_{m-1}-1$ distribution of zeros if $i_{1} \leqq j_{1}$.

In $\$ 3$ we deduce sufficient conditions for nonexistence of extremal solutions with certain zero distributions. These conditions are given in terms of inequalities imposed on the coefficients of (1.1).

2. Distribution of zeros. Let $y_{1}, y_{2}, \cdots, y_{n}$ be $n$ linearly independent solutions of (1.1). Define

$$
\begin{aligned}
& w\left(x ; x_{1}^{\left[k_{1}\right]}, x_{2}^{\left[k_{2}\right]}, \cdots, x_{p}^{\left[k_{k}\right]}\right) \\
& =\left|\begin{array}{cccc}
y_{1}(x) & y_{2}(x) & \cdots & y_{n}(x) \\
y_{1}\left(x_{1}\right) & y_{2}\left(x_{1}\right) & \cdots & y_{n}\left(x_{1}\right) \\
y_{1}^{\prime}\left(x_{1}\right) & y_{2}^{\prime}\left(x_{1}\right) & \cdots & y_{n}^{\prime}\left(x_{1}\right) \\
& \cdots & & \\
y_{1}^{\left(k_{1}-1\right)}\left(x_{1}\right) & y_{2}^{\left(k_{1}-1\right)}\left(x_{1}\right) & \cdots & y_{n}^{\left(k_{1}-1\right)}\left(x_{1}\right) \\
y_{1}\left(x_{2}\right) & y_{2}\left(x_{2}\right) & \cdots & y_{n}\left(x_{2}\right) \\
& \cdots & & \\
y_{1}\left(x_{p}\right) & y_{2}\left(x_{p}\right) & \cdots & y_{n}\left(x_{p}\right) \\
& \cdots & & \\
y_{1}^{(k, p-1)}\left(x_{p}\right) & y_{2}^{\left(k_{p}-1\right)}\left(x_{p}\right) & \cdots & y_{n}^{\left(k_{p}-1\right)}\left(x_{p}\right)
\end{array}\right|,
\end{aligned}
$$

$1 \leqq p \leqq n-1, k_{1}+k_{2}+\cdots+k_{x}=n-1$.

From (2.1) and the continuous dependence of $y_{1}, y_{2}, \cdots, y_{n}$ and their derivatives on $x_{1}, x_{2}, \cdots, x_{p}$, we easily obtain the following lemma:

LEMMA 2.1. For fixed $x_{i}, i=1,2, \cdots, p, w(x)=w\left(x ; x_{1}^{\left[k_{2}\right]}, \cdots, x_{p}^{\left[k_{p}\right]}\right)$ is a solution of (1.1) with a zero of order $k_{i}$ at $x_{i}, i=1,2, \cdots, p$. Moreover, 
it is a continuous function of $x_{1}, \cdots, x_{p}$ on $I$, i.e., for a given $\epsilon>0$ there exists a $\delta=\delta\left(x_{1}, \cdots, x_{p}\right)>0$ such that

$$
\left|w\left(x ; x_{1}^{\left[k_{1}\right]}, \cdots, x_{p}^{\left[k_{p}\right]}\right)-w\left(x ; \xi_{1}^{\left[k_{1}\right]}, \cdots, \xi_{p}^{\left[k_{p}\right]}\right)\right|<\epsilon,
$$

$x \in[a, b] \subset I, b-a<\infty$, provided $\sum_{i=1}^{p}\left|x_{i}-\xi_{i}\right|<\delta$.

THEOREM 2.1. Suppose that (1.1) has an extremal solution $y$ for $\left[\alpha, \eta_{1}(\alpha)\right]$ with an $i_{1}-i_{2}-\cdots-i_{l}$ distribution of zeros, $i_{1}+i_{2}+\cdots+$ $i_{l}=n$. If Y has a zero of order exactly $i_{m}$ at $x_{m}, 2 \leqq m \leqq l-1$, then (1.1) has an extremal solution for $\left[\alpha, \eta_{1}(x)\right]$ with an $i_{1}-\cdots-i_{m-1}$ $\left(i_{m}-1\right)-i_{m+1}-\cdots-i_{l}$ distribution of zeros and an additional zero at an arbitrary point $\xi \in\left[\alpha, \eta_{1}(\alpha)\right]$.

Proof. Since $2 \leqq m \leqq l-1$, we may assume $l \geqq 3$. By the hypotheses, we have $y\left(x_{k}\right)=y^{\prime}\left(x_{k}\right)=\cdots=y^{\left(i_{k}-1\right)}\left(x_{k}\right)=0, k=1,2$, $\cdots, l$, and $\mathrm{y}^{\left(i_{m}\right)}\left(x_{m}\right) \neq 0, \alpha=x_{1}<x_{2}<\cdots<x_{l}=\eta_{1}(\alpha)$. Consider the function

$$
w(x)=w\left(x ; x_{1}^{\left[i_{1}\right]}, \cdots, x_{m-1}^{\left[i_{m-1}\right]}, x_{m}^{\left[i_{m}-1\right]}, x_{m+1}^{\left[i_{m+1}\right]}, \cdots, x_{l}^{\left[i_{i}\right]}\right)
$$

defined in (2.1). The existence of $y$ requires $w^{\left(i_{m}-1\right)}\left(x_{m}\right)=0$. Therefore, $w$ is an extremal solution of $(1.1)$ for $\left[\alpha, r_{1}(\alpha)\right]$ with an $i_{1}-i_{2}-\cdots-i_{l}$ distribution of zeros, unless it vanishes identically. We assert that either $w$ vanishes identically or $w \not \equiv 0, w^{\left(i_{m}\right)}\left(x_{m}\right)=0$. For proof assume the contrary: $w \neq \equiv 0$ and $w^{\left(i_{m}\right)}\left(x_{m}\right) \neq 0$. Then $w$ is an extremal solution with an $i_{1}-i_{2}-\cdots-i_{i}$ distribution of zeros. Moreover, $w$ has a zero of order exactly $i_{m}$ at $x_{m}$. Hence, there exists $a \delta>0$ such that

$$
w_{\delta}(x)=w\left(x ; x_{1}^{\left[i_{1}\right]}, \cdots, x_{m-1}^{\left[i_{m-1}\right]}, x_{m}^{\left[i_{m-1}\right]}, x_{m+1}^{\left[i_{m+1}\right]}, \cdots,\left(x_{l}-\delta\right)^{\left[i i^{\prime}\right]}\right)
$$

is a nontrivial solution satisfying

$$
\left|n(x)-w_{\delta}(x)\right|<\epsilon, \quad x \in\left[x, \eta_{1}(x)\right],
$$

for a given $\epsilon>0$ by Lemma 2.i. Evidently, this solution has the following $n-1$ zeros on $\left[x, \eta_{1}(x)-\delta\right]$ : a zero of order $i_{k}$ at $x_{k}, k=1, \cdots, m-1$, $m+1, \cdots, l-1$, a zero of order $i_{m}-1$ at $x_{m}$, and a zero of order $i_{l}$ at $x_{l}-\delta$. Furthermore, for an arbitrarily small $\epsilon_{1}>0$, there exists $w_{\delta}$ such that either $w_{\delta}^{\left(i_{m}-1\right)}\left(x_{m}\right)=0$ or $w_{\delta}(\zeta)=0$ for some point $\zeta,\left|\zeta-x_{m}\right|<$ $\epsilon_{1}$. This follows from (2.2) for a sufficiently small $\epsilon$, since the zeros can disappear only in pairs. In either case, $w_{\delta}$ would have $n$ zeros on $\left[\alpha, \eta_{1}(\alpha)-\delta\right], \delta>0$, contrary to the definition of $\eta_{1}(\alpha)$. This contradiction proves the assertion that either $w \equiv 0$, or $w \neq 0$ and $w^{\left(i_{m}\right)}\left(x_{m}\right)=$ 0 . Suppose first that $w \equiv 0$. In this case, $w(\xi)=0$ for every $\xi \in\left[x, \eta_{1}(\alpha)\right]$. However, this is a sufficient condition for the existence of a nontrivial solution of (1.1) with a zero at $\xi$, a zero of order $i_{k}$ at $x_{k}, k=1, \cdots$, 
$m-1, m+1, \cdots, l$, and a zero of order $i_{m}-1$ at $x_{m}$. When $\xi$ coincides with one of the zeros, say, $\xi=x_{q}, 1 \leqq q \leqq l$, a similar result follows from $w^{\left(i_{0}\right)}(\xi)=0$. Finally, we consider the case $w \neq 0, w^{\left(i_{m}\right)}\left(x_{m}\right)=0$. Since $y^{\left(i_{m}\right)}\left(x_{m}\right) \neq 0, y$ and $w$ are two linearly independent extremal solutions with a zero of order $i_{k}$ at $x_{k}, k=1,2, \cdots, i$. Hence, there exists a nontrivial linear combination of $y$ and $w$ with a zero at an arbitrary point $\xi$ and a zero of order $i_{\kappa}$ at $x_{k}, k=1,2, \cdots, l$. This completes the proof.

If (1.1) has an extremal solution $u$ with an $i_{1}-i_{2}-\cdots-i_{l-1}-1$ distribution of zeros, $i_{1}+i_{2}+\cdots+i_{l-1}=n-1$, then

$$
u\left(x_{i k}\right)=u^{\prime}\left(x_{k}\right)=\cdots=u^{\left(i_{k}-1\right)}\left(x_{k}\right)=0, \quad u^{\left(i_{k}\right)}\left(x_{k}\right) \neq 0,
$$

$k=1,2, \cdots, l-1$. Consequently, by a repeated application of Theorem 2.1 , we obtain the following results.

THEOREM 2.2. Suppose (1.1) has an extremal solution for $\left[\alpha, \eta_{1}(\alpha)\right]$ with an $i_{1}-i_{2}-\cdots-i_{l-1}-1$ distribution of zeros, $i_{1}+i_{2}+\cdots+$ $i_{l-1}=n-1$. Let $j_{1}, j_{2}, \cdots, j_{m-1}, 2 \leqq m \leqq n$, be arbitrary positive integers such that $j_{1}+j_{2}+\cdots+j_{m-1}=n-1$, and let $\xi_{2}, \xi_{3}, \cdots, \xi_{m-1}$ be arbitrary distinct points on $\left(\alpha, \eta_{1}(\alpha)\right)$. If $i_{1} \leqq j_{1}$, (1.1) has an extremal solution for $\left[\alpha, \eta_{1}(\alpha)\right]$ which has a zero of order exactly $j_{1}$ at $\alpha$, a zero of order exactly $j_{k}$ at $\xi_{k}, k=2,3, \cdots, m-1$, and a zero at $\eta_{1}(\alpha)$.

We now easily deduce the following statements from Theorems 2.1 and 2.2 .

THEOREM 2.3. Suppose (1.1) has an extremai solution for $\left[\alpha, \eta_{1}(\alpha)\right]$ with an $i_{1}-i_{2}-\cdots-i_{l-1}-1$ distribution of zeros, $i_{1}+i_{2}+\cdots+i_{l-1}=$ $n-1$. For any integer $m, 2 \leqq m \leqq n-1$, let $h_{1}, h_{2}, \cdots, h_{m-1}$ be arbitrary positive integers such that $h_{1}+h_{2}+\cdots+h_{m-1}=n-2$, and let $\xi_{2}, \xi_{3}, \cdots, \xi_{m-1}$ be arbitrary distinct points on $\left(\alpha, \eta_{1}(\alpha)\right)$. If $i_{1} \leqq h_{1}$, (1.1) has an extrema! solution for $\left[x, \eta_{1}(\alpha)\right]$ which has a zero of order $h_{1}$ at $\alpha$, a zero of order $h_{k}$ at $\xi_{k}, k=2,3, \cdots, m-1$, and a double zero at $v_{i_{1}}(\alpha)$.

Next we consider an extremal solution $y$ with a $1-i_{2}-\cdots-i_{t}$ distribution of zeros. According to a result of Sherman [14], the first conjugate point $\eta_{1}(x)$ is a strictly increasing function of $\alpha$. It follows from this that $y\left(x_{k}\right)=y^{\prime}\left(x_{k}\right)=\cdots=y^{\left(i_{k}-1\right)}\left(x_{k}\right)=0, y^{\left(i_{k}\right)}\left(x_{k}\right) \neq 0, k=2$, $3, \cdots, l$. Therefore, in this case we have results similar to Theorems 2.1, 2.2, and 2.3, which we state in the following theorem.

THEOREM 2.4. Suppose (1.1) has an extremal solution for $\left[\alpha, \eta_{1}(\alpha)\right]$ with a $1-i_{2}-\cdots-i_{l}$ distribution of zeros, $i_{2}+i_{3}+\cdots+i_{l}=n-1$. 
Let $j_{2}, j_{3}, \cdots, j_{m}, 2 \leqq m \leqq n$, be arbitrary positive integers such that $j_{2}+j_{3}+\cdots+j_{m}=n-1$, and let $\xi_{2}, \cdots, \xi_{m-1}$ be arbitrary distinct points on $\left(\alpha, \eta_{1}(\alpha)\right)$. If $i_{\imath} \leqq j_{m},(1.1)$ has an extremal solution for $\left[\alpha, \eta_{\mathbf{1}}(\alpha)\right]$ which has a zero at $\alpha$, a zero of order exactly $j_{k}$ at $\xi_{k}, k=2,3, \cdots, m-1$, and a zero of order exactly $j_{m}$ at $\eta_{1}(\alpha)$.

Similarly, if $h_{2}, h_{3}, \cdots, h_{r}, 2 \leqq r \leqq n-1$, are arbitrary positice integers such that $h_{2}+h_{3}+\cdots+h_{r}=n-2$ and $i_{l} \leqq h_{r}$, then (1.1) has an extremal solution for $\left[\alpha, \eta_{1}(\alpha)\right]$ with a $2-h_{2}-\cdots-h_{r}$ distribution of zeros. The zeros occur at the prescribed points on $\left(\alpha, \eta_{1}(\alpha)\right)$.

Our next result is concerned with the existence of an extremal solution with simple zeros on $\left(\alpha, \eta_{1}(\alpha)\right)$.

THEOREM 2.5. Suppose (1.1) has an extremal solution for $\left[\alpha, \eta_{1}(\alpha)\right]$ with an $i_{1}-i_{2}-\cdots-i_{l}$ distribution of zeros, $i_{1}+i_{2}+\cdots+i_{l}=\eta$. Then (1.1) has an extremal solution $\mathrm{Y}_{\text {for }}\left[\alpha, \eta_{1}(\alpha)\right]$ such that

(i) every zero of $y$ on $\left(\alpha, \eta_{1}(\alpha)\right)$ is simple,

(ii) $\mathcal{Y}$ has at least $i_{2}+\cdots+i_{l-1}$ distinct zeros on $\left(\alpha, \eta_{1}(\alpha)\right)$.

Proof. We shall first show that there exists an extremal solution which has at least $i_{2}+\cdots+i_{l-1}$ distinct zeros on $\left(\alpha, \eta_{1}(\alpha)\right)$. Let $y_{1}$ be an extremal solution with an $i_{1}-i_{2}-\cdots-i_{l}$ distribution of zeros. Then $y_{1}$ has at least $l-2$ distinct zeros on $\left(\alpha, \eta_{1}(\alpha)\right)$. Without loss of generality, we may assume that $y_{1}$ has a zero of order exactly $i_{k}$ at $x_{k} \in\left(\alpha, \eta_{1}(\alpha)\right), k=2,3, \cdots, l-1$. If $i_{k}=1, k=2,3, \cdots, l-1$, there is nothing to prove. Hence, we shall assume $i_{p} \geqq 2$ for some $p$, $2 \leqq p \leqq l-1$. Then, by Theorem $2.1,(1.1)$ has an extremal solution $y_{2}$ with an $i_{1}-\cdots-i_{p-1}-\left(i_{n}-1\right)-i_{p+1}-\cdots-i_{l}$ distribution of zeros, and an additional zero at a point $\xi \in\left[x, \eta_{1}(x)\right], \xi \neq x_{k}, k=1$, $2, \cdots, l$. Clearly, $y_{2}$ has at least $l-1$ distinct zeros on $\left(\alpha, \eta_{1}(\alpha)\right)$. A successive application of the above argument will establish the existence of an extremal solution which has at least $i_{2}+\cdots+i_{l-1}$ distinct zeros on $\left(x, \eta_{1}(x)\right)$. Let $y$ be an extremal solution which has the largest number $N$ of distinct zeros on $\left(\alpha, \eta_{1}(\alpha)\right), n-2 \geqq N \geqq i_{2}+\cdots+i_{l-1}$. All the zeros of $y$ on $\left(\alpha, \eta_{1}(\alpha)\right)$ must be simple; otherwise, we can repeat the previous argument and establish the existence of an extremal solution with at least $N+1$ distinct zeros on $\left(\alpha, \eta_{1}(\alpha)\right)$, contrary to the choice of $N$.

3. Sufficient conditions. There are known conditions under which (1.1) has no nontrivial solution with an $(n-1)-1$ or $1-(n-1)$ distribution of zeros. No nontrivial solution of $(1.1)$ has an $(n-1)-1$ distribution of zeros if $p_{i} \leqq 0, i=0,1, \cdots, n-1[6],[13]$. On the other hand, if $(-1)^{n-i} p_{i} \leqq 0, i=0,1, \cdots, n-1$, then no nontrivial solution of (1.1) has a $1-(n-1)$ distribution of zeros [13]. We can improve 
these results by noting that the conditions on $p_{n-1}$ may be dropped. Equation (1.1) can first be multiplied by $\exp \left\{\int p_{n-1} d x\right\}$ to get

$$
\begin{aligned}
{\left[\exp \left\{\int p_{n-1} d x\right\} y^{(n-1)}\right]^{\prime}+\exp \left\{\int p_{n-1} d x\right\} p_{n-2} y^{(n-2)} } & \\
& +\cdots+\exp \left\{\int p_{n-1} d x\right\} p_{0} y=0,
\end{aligned}
$$

to which the proofs given in [6], [13] can be applied.

From these results and Theorem 2.2, we obtain the following statements.

THEOREM 3.1. If $p_{i} \leqq 0, i=0,1, \cdots, n-2$, then every extremal solution of (1.1) for $\left[\alpha, \eta_{1}(\alpha)\right]$ has a zero of order 2 at $\eta_{1}(\alpha)$ and has no more than $n-2$ zeros on $\left[\alpha, \eta_{1}(\alpha)\right)$. Similarly, if $(-1)^{n-i} p_{i} \leqq 0, i=0,1, \cdots$, $n-2$, then every extremal solution of (1.1) for $\left[\alpha, \eta_{1}(\alpha)\right]$ has a zero of order 2 at $\alpha$ and has no more than $n-2$ zeros on $\left(\alpha, \eta_{1}(\alpha)\right]$.

According to a result of Leighton and Nehari [8], the selfadjoint equation

$$
\left(r y^{\prime \prime}\right)^{\prime \prime}-p y=0, \quad r>0, p>0, r \in C^{\prime \prime}, p \in C,
$$

has an extremal solution for $\left[\alpha, \eta_{1}(\alpha)\right]$ which does not vanish on $\left(\alpha, \eta_{1}(\alpha)\right)$ and which has zeros of order exactly 2 at $\alpha$ and $\eta_{1}(\alpha)$, provided that (3.1) has a nontrivial solution which vanishes at $\alpha$ and which has at least four zeros on $[\alpha, \infty)$. In this connection we make the following observation. If $p_{0} \leqq 0, p_{2} \leqq 0$, and $p_{3}$ are continuous on an interval $I$, every extremal solution for $\left[\alpha, \eta_{1}(\alpha)\right] \subset I$ of

$$
y^{(4)}+p_{3} y^{\prime \prime \prime}+p_{2} y^{\prime \prime}+p_{0} y=0
$$

has zeros of order exactly 2 at $\alpha$ and $\eta_{1}(\alpha)$, and does not vanish on $\left(\alpha, \eta_{1}(\alpha)\right)$. This is immediate from Theorem 3.1.

\section{REFERENCES}

1. R. G. Alicv, On certain properties of solutions of ordinary differential equations of fourth order, Sb. Aspirantsh. Kazan Institute 1964, 15-30. (Russian)

2. N. V. Azbelev and Z. B. Caljuk, On the question of distribution of zeros of solutions of linear differential equations of third order, Mat. Sb. 51 (93) (1960), 475-486; English transl., Amer. Math. Soc. Transl. (2) 42 (1964), 233-245. MR 22 \#12266.

3. J. H. Barrett, Third-order differential equations with nonnegative coefficients, J. Math. Anal. Appl. 24 (1968), 212--224. MR 38 \#365.

4. - Oscillation theory of ordinary linear differentiai equations, Advances in Math. 3 (1969), 415-509. MR 41 \#2113.

5. P. Hartman, Unrestricted n-parameter families, Rend. Circ. Mat. Palermo (2) 7 (1958), 123-142. MR 21 \#4211.

6. W. J. Kim, Oscillatory properties of linear third-order differential equations, Proc. Amer. Math. Soc. 26 (1970), 286-293. 
7. W. J. Kim, Simple zeros of solutions of nth-order linear differential equations, Proc. Amer. Math. Soc. 28 (1971), 557-561.

8. W. Leighton and $Z$. Nehari, On the oscillation of solutions of self-adjoint linear differential equations of the fourth order, Trans. Amer. Math. Soc. 89 (1958), 325-377. MR 21 \#1429.

9. A. Ju. Levin, The non-oscillation of solutions of the equation $x^{(n)}+p_{1}(t) x^{(n-1)}+$ $\cdots+p_{n}(t) x=0$, Uspehi Mat. Nauk 24 (1969), no. 2 (146), 43-96=Russian Math. Surveys 24 (1969), no. 2, 43-99. MR 40 \#7537.

10. Z. Opial, On a theorem of $O$. Aramă, J. Differential Equations 3 (1967), 88-91. MR 34 \#6194.

11. A. C. Peterson, Distribution of zeros of solutions of a fourth order differential equation, Pacific J. Math. 30 (1969), 751-764. MR 40 \#5975.

12. —, A theorem of Aliev, Proc. Amer. Math. Soc. 23 (1969), 364-366. MR 40 \#2961.

13. V. Pudei, Über die Eigenschaften der Lösungen linearer Differentialgleichungen gerader Ordnung, Casopis Pěst. Mat. 94 (1969), $401-425$.

14. T. L. Sherman, Properties of solutions of nth order linear differential equations, Pacific J. Math. 15 (1965), 1045-1060. MR 32 \#2654.

15. - Conjugate points and simple zeros for ordinary linear differential equations, Trans. Amer. Math. Soc. 146 (1969), 397-411. MR 41 \#572.

Department of Applied Mathematics and Statistics, State University of New YORK AT Stony Brook, Stony broOK, NeW York 11790 